\title{
Trabalho em grupo: a percepção do profissional do sistema único saúde*
}

\author{
Group Work: Perception of the Professional Health System User
}

Trabajo en grupo: la percepción del profesional del sistema único de salud

Valéria Cristina Dos Santos Carvalho

Universidade Paulista, Brasil

vaegab2@hotmail.com

ORCID: http://orcid.org/0000-0001-6724-2192

Antonio Carlos Siqueira Junior

Faculdade de Medicina de Marilia-Famema, Brasil

ORCID: http://orcid.org/0000-0003-2351-6256

Fernanda Paula Cerântola Siqueira

Faculdade de Medicina de Marília, Brasil ORCID:

http://orcid.org/0000-0002-9331-7685
DOI: https://doi.org/10.11144/Javeriana.ie21-1.tgpp Redalyc: http://www.redalyc.org/articulo.oa? id $=145257605010$

Recepção: 06 Setembro 2016 Aprovação: 23 Outubro 2018

\section{Resumo:}

Introdução: O trabalho em grupo no Sistema Único de Saúde é utilizado pelos profissionais como estratégia de promoção, prevenção e reabilitação da saúde, o qual proporciona a construção de práticas fortalecidas pela necessidade coletiva. Objetivo: Identificar a percepção dos profissionais que atuam em serviços do Sistema Único Saúde-SUS sobre o atendimento em grupo. Método: Estudo de abordagem qualitativa, realizado num município de pequeno porte da região sudeste do Brasil, tendo como sujeitos da pesquisa profissionais que realizaram atividades em grupo e usuários do Sistema Único de Saúde que participaram de algum grupo oferecido pela Unidade de Saúde selecionada. Os dados foram coletados por meio de entrevista gravada e aberta com questões norteadoras para o profissional e para o usuário. Para análise dos dados, utilizou-se técnica de análise do conteúdo de Bardin na modalidade temática. Resultados: Foram entrevistados nove profissionais de saúde que compõem a equipe multiprofissional. Identificaram as seguintes categorias temáticas: 1) a compreensão sobre grupo: conceito, habilidades necessárias e benefícios. 2) A prática grupal como estratégia educativa e organizacional, e 3) dificuldades: pouca adesão e despreparo profissional para desenvolver as atividades de grupo. Conclusão: Os profissionais sentem-se despreparados e apontam a necessidade de aprimorar seu conhecimento para desenvolver a prática grupal. Percebem que, quando coordenado por um profissional com aproximação das técnicas grupais, o grupo torna-se um recurso valioso para a promoção de saúde, para a construção de novos conhecimentos e para o fortalecimento do vínculo entre o professional e usuário.

Palavras-chave: processos grupais, sistema único de saúde, percepção social.

\begin{abstract}
:
Introduction: the professionals use the group work in the Unified Health System as a strategy for the promotion, prevention and rehabilitation of health, which provides the construction of practices strengthened by the collective need. Objective: To identify the perception of the professionals who work in SUS-SUS services on group care. Method: A qualitative approach was carried out in a small municipality in the Southeast region of Brazil, having as research subjects professionals who performed activities in a group and users of the Unified Health System who participated in a group offered by the selected Health Unit. Data were collected through interviews and analyzed using Content Analysis in the Thematic Mode proposed by Bardin. Results: We interviewed nine health professionals that make up the multiprofessional team. The following thematic categories were identified: (1) Understanding about group meaning: concept, necessary skills and benefits; (2) the group practice as an educational and organizational strategy, and (3) difficulties: little adherence and no professional preparation to develop group activities. Conclusion: The professionals, despite feeling unprepared, realize that the group is a valuable resource for health promotion, construction of new knowledge and for strengthening the bond between professional and user.
\end{abstract}

Keywords: group processes, unified health system, social perception. 


\section{Resumen:}

Introducción: El trabajo en equipo en el Sistema Único de Salud (SUS) lo usan profesionales como estrategia de promoción, prevención y rehabilitación de la salud, pues proporciona prácticas fortalecidas por la necesidad colectiva. Objetivo: Identificar la percepción de los profesionales que actúan en servicios del SUS sobre la atención en grupo. Método: Estudio de abordaje cualitativo realizado en un municipio de pequeño porte de la región sudeste de Brasil, teniendo como sujetos de la investigación a profesionales que han realizado actividades en grupo y usuarios del SUS que han participado de algún grupo ofrecido por la unidad de salud seleccionada. Los datos se recogieron mediante entrevistas y se detallaron con el Análisis de Contenido en la Modalidad Temática, propuesto por Bardin. Resultados: Se entrevistaron nueve profesionales en salud que componen el equipo multidisciplinario. Se identificaron las siguientes categorías temáticas: 1) la comprensión sobre el grupo: concepto, habilidades necesarias y beneficios; 2) la práctica en grupo como estrategia educativa y organizacional, y 3) las dificultades: poca adhesión y falta de preparación profesional para desarrollar las actividades en grupo. Conclusión: Aunque los profesionales no se sientan preparados, perciben que el grupo es un recurso valioso para la promoción de la salud, la construcción de nuevos conocimientos y el fortalecimiento del vínculo entre profesional y el usuario.

Palabras clave: procesos de grupo, sistema único de salud, percepción social.

\section{Introdução}

Os grupos no Sistema Único de Saúde (SUS) são utilizados no processo de cuidado à saúde e à doença como estratégias para promoção, prevenção e reabilitação da saúde por incluírem a pessoa as decisões do tratamento, e fazê-la comprometer-se em criar ações na vida diária que possam gerar saúde (1).

$\mathrm{Na}$ atenção primária, os grupos têm sido utilizados como proposta de cuidar buscando constantemente alcançar o princípio da integralidade, a autonomia do sujeito e a troca das sensações e sentimentos vivenciados. Tais ações têm o objetivo de garantir a participação popular e a construção de práticas fortalecidas pela necessidade coletiva e subjetiva (2).

Os profissionais de saúde, principalmente os da atenção primária, têm fácil acesso às residências das pessoas, podendo entender suas angústias, seus problemas, sua vivência, cultura e, principalmente, o processo de adoecimento biopsicossocial (3). Os processos grupais existentes no SUS são utilizados para diversos propósitos: na reabilitação e tratamento das doenças crônicas, como os grupos de pessoas com diabetes mellitus ou hipertensão arterial sistêmica; no cuidado às gestantes e seu corpo, com as crianças e suas mães; com os idosos para entenderem o processo do envelhecimento; com adolescentes e jovens no cuidado com as doenças sexualmente transmissíveis, entre outros assuntos que necessitam ser abordados (4).

Estudos brasileiros apontam que as atividades em grupos educativos são voltadas para a adesão ao tratamento propostos pelos programas do Ministério da Saúde e na prevenção de riscos e agravos, especialmente nas doenças crônicas; sendo, assim, organizados com reunião de pessoas para assistir a uma palestra $(5,6)$. Observa-se que essa forma de realizar as atividades em grupo seja decorrentes da falta de preparo, de planejamento, de capacitação e de conhecimento dos profissionais acerca de como realizar um grupo educativo e todo o processo de dinâmica de grupos (7).

Muitas vezes, empiricamente observa-se que a construção e a efetivação do grupo ocorrem sem preparo prévio, sem uma metodologia norteadora e, até mesmo, sem um local adequado para realizar as atividades. É identificado que, em linhas gerais, essa prática ocorre apenas como cumprimento de etapas previstas e requeridas por gestores, havendo preocupação com a proposta principal do grupo. 
Tais aspectos despertaram a necessidade desta investigação, a qual surgiu a partir da vivência da prática grupal dos próprios autores ao identificarem lacunas na realização dos grupos, nos papéis desenvolvidos e na pouca clareza de que o convívio grupal é um instrumento de grande valia.

Considerando a problemática ainda existente em relação à prática grupal esta pesquisa objetivou identificar a percepção dos profissionais que atuam em serviços do Sistema Único Saúde-SUS sobre o atendimento em grupo.

\section{Considerações de método}

Apoiando-se na natureza do objeto a ser investigado, optou-se por desenvolver uma pesquisa exploratória de abordagem qualitativa, a qual busca compreender e explorar os conceitos concebidos ao longo da vida, as emoções humanas, os comportamentos, experiências e as ações dos sujeitos no próprio contexto de vida (8).

O cenário desta pesquisa foi composto por nove unidades de saúde pertencentes a atenção básica de um município de pequeno porte da região sudeste do Brasil. A escolha do referido cenário justificouse pelo fato de esse ser considerado o local em que mais há desenvolvimento de grupos. É na atenção básica que os grupos de trabalho são promovidos e conduzidos por múltiplos profissionais. Sendo assim, profissionais participam juntos, realizam cuidados de promoção, prevenção, diagnóstico, tratamento e reabilitação da pessoa procurando utilizar práticas gerenciais, sanitárias, democráticas e participativas da equipe multiprofissional, seguindo sempre os princípios do SUS.

Foram selecionados nove profissionais, sendo um de cada unidade do referido cenário deste estudo eutilizou-se como critério de inclusão ser um dos profissionais de saúde da equipe multiprofissional, ter alguma experiência no desenvolvimento de grupos e aceitar participar da pesquisa.

Para a coleta de dados, utilizou-se a técnica da entrevista semiestruturada, na qual o participante teve a possibilidade de discorrer sobre suas experiências por meio das seguintes questões norteadoras: Como você compreende grupo e como desenvolve seu trabalho? Como vocês compreendem os papéis grupais? Qual a metodologia utilizada para o planejamento do seu grupo de trabalho?

As entrevistas foram realizadas no período de junho a setembro de 2014 , foram gravadas, transcritas na íntegra e analisadas, utilizando-se a técnica de análise de conteúdo na modalidade temática proposta por Bardin. A análise do conteúdo visa obter, por procedimentos sistemáticos e objetivos as condições de produção/recepção (variáveis inferidas) das falas dos sujeitos participantes, percorrendo as etapas de préanálise primeira fase, sendo o momento da organização, da leitura e da revisão do material colhido pelo pesquisador com observações particulares, visão do todo e propostas iniciais para o análise e interpretação. Na segunda etapa, ocorre a exploração desse material, estabelecendo-se regras pontuais nos dados do texto, junção, enumeração e apresentação do conteúdo de forma simplificada. A terceira etapa tem como objetivo, ler os fragmentos do texto categorizado, discutir partes do texto, articulando-as a conceitos propostos na teoria, para realizar a análise e buscar as unidades de significação ou categoria articuladas no desenvolvimento do conceito teórico percorrido pela análise (9).

$\mathrm{O}$ desenvolvimento da pesquisa atendeu às normas nacionais e internacionais de ética em pesquisa envolvendo seres humanos. O projeto foi apreciado pelo Comitê de Ética em Pesquisa envolvendo seres humanos da Faculdade de Medicina de Marília (Famema) e aprovado sob o número CAAE: 26241314.3.0000.5413 e número do parecer 539.514. E para garantir o anonimato dos nove profissionais participantes utilizou-se como códigos a letra "P" acompanhada das letras minúsculas que identifica as respectivas categorias profissionais, sendo "e" para enfermeiro, "p" para psicólogo e "ef” para educador físico, seguidos ainda de numeração, como, por exemplo "Pe1", "Pp1", "Pef1", sucessivamente. 


\section{Resultados/discussão}

\section{Caracterização dos sujeitos da pesquisa}

Foram entrevistados nove profissionais de saúde, entre os quais uma enfermeira assistencial e coordenadora de Unidade Básica de Saúde (UBS); quatro enfermeiras coordenadoras e assistentes da Estratégia de Saúde da Família (ESF); três psicólogos atuantes em UBS, e um educador físico. Esses profissionais, com formações específicas, realizavam grupos nas UBS do seu território. Em relação ao tempo de formação os profissionais possuíam sete anos ou mais.

\section{Apresentação das categorias temáticas}

\section{Categoria 1. A compreensão sobre grupo: conceito, habilidades necessárias e beneficios}

Esta categoria expressa as diferenças na compreensão do conceito de grupo, bem como a forma de realização da prática grupal e seus benefícios nos cenários deste estudo.

"É a forma comum de juntar pessoas [...]" (Pp8). "[...] entendo esse grupo como um espaço onde as pessoas possam falar e serem ouvidas [...]" (Pe6). "[...] é quando vários indivíduos têm a mesma necessidade e, trabalhando em grupo, você consegue, através da experiência dos outros, desenvolver mais este assunto [...], tanto para o paciente como para a equipe" (Pp3).

Os profissionais participantes desta pesquisa conceituaram grupo como reunião de pessoas, espaço para diálogo e troca de experiência que, reconhecidamente, estão presentes no conceito, mas os elementos descritos isoladamente não sustentam a definição de grupo, pois esta avança para a necessidade de um objetivo comum.

De acordo com Zimerman e Osório, num grupo as pessoas tendem a construir juntas um objetivo comum, quando observamos uma reunião ou um agrupamento as características apresentadas não são resolutivas, apresentam falas técnicas nem sempre adequadas a realidade de quem está ouvindo tornando se assim fragmentada e sem continuidade (10).

Dessa forma, o planejamento de um grupo deve emergir a partir de uma avaliação inicial das necessidades e demandas dos sujeitos da população a ser trabalhada. Deve-se levar em consideração alguns aspectos importantes, os primeiros são, a saber, as características dos participantes do grupo e quem serão eles (5).

Para tanto, os participantes reconhecem que para realizar a atividade grupal é necessário desenvolver habilidades como empatia, escuta, troca efetiva e relacionamento interpessoal com os participantes do grupo. "[...] paciência para inserir essa pessoa no grupo. A pessoa, nessa idade, tem muita dificuldade de inserção nos grupos e eu tenho minha virtude, que é essa: tenho uma facilidade de relacionamento no grupo" (Pef5). [...] você tem que se abrir para a escuta, porque, aí, você tem uma troca” (Pef5). A forma de conduzir o grupo, ou seja, a metodologia, deve permitir a participação, a interação das pessoas, reflexão sobre as questões levantadas e troca de experiências (11).

Além disso, o profissional, nas atividades em grupos, deve ser capaz de instrumentalizar os integrantes, estimulando o desenvolvimento da consciência crítica e o exercício da autonomia para as decisóes de saúde (12). Para tanto, é necessário que os profissionais que coordenem o grupo tenham atributos como: gostar e acreditar em grupos; ter paciência, empatia, senso de ética, respeito, senso de humor, capacidade de comunicação; ser verdadeiro e ter capacidade de integração e síntese (10).

Essas habilidades são apontadas como essenciais para um trabalho efe tivo em grupo, requer uma perspectiva de relação entre as pessoas que são percebidas como sujeitos singulares e responsáveis por suas próprias vidas e não apenas objetos de intervenção. Desta forma, a atividade de grupo interfere na prática de cuidado e na percepção biopsicossocial (13). 
Os participantes expressam em seus relatos que um dos benefícios que a atividade grupo proporciona é o fortalecimento do vínculo. “[...] o vínculo fica maior com o grupo" (Pef5); “[...] o vínculo entre elas e nós profissionais é de amizade e entre elas e vizinhos da própria rua” (Pe4).

Em outros cenários da atenção primária em saúde, os grupos são considerados espaços privilegiados para o desenvolvimento das atividades educativas na saúde e têm representado uma alternativa para as práticas assistenciais que favorecem uma maior participação e construção do vínculo (14).

Pesquisas recentes destacam os benefícios advindos do convívio em grupo, mas ressaltam também a necessidade de estar atento a problemas que possam surgir e serem superados pelos mesmos, como dificuldade em colocar-se no grupo, respeito mútuo, dificuldade de concentração, sair do objetivo proposto. Mas acredita-se que quando o grupo encontra-se numa vivência constante esses fatores são discutidos e resolvidos possibilitando também um aprendizado mutuo $(15,16)$.

Categoria 2. A prática grupal como estratégia educativa e organizacional

Os profissionais reconhecem que a atividade em grupo é importante, gratificante e produtiva pela possibilidade de atingir um maior número de pessoas e por favorecer o aprendizado dos indivíduos, principalmente daqueles mais introvertidos. “[...] acho muito importante o trabalho do grupo" (Pe2); “[...] eu gosto de realizar grupo, porque o grupo você abrange várias pessoas e acaba tirando dúvidas de algumas pessoas que nem sempre vão perguntar [...] além de eu achar gratificante, eu acho ele produtivo" (Pp1).

As ações educativas desenvolvidas pelos enfermeiros precisam ser planejadas e implementadas com a utilização de diversas estratégias metodológicas para nortear a prática efetiva, sendo permeadas pela valorização do diálogo, pela troca de experiências e pelo respeito à cultura dos sujeitos $(17,18)$.

Entre os tipos de grupos desenvolvidos pelos profissionais participantes deste estudo, destaca-se aqueles com o objetivo de ação educativa. "[...] na nossa unidade funcionam vários grupos [...] são grupos de orientação, acolhimento e troca de informações [...]” (Pe6).

$\mathrm{Na}$ fala dos participantes aflora o que o Ministério da saúde preconiza como atribuições comuns entre os profissionais das equipes que atuam na Atenção Primária, como a organização de grupos e atividades de educação para a saúde, as quais visam à promoção, à proteção e à recuperação da saúde, com uma abordagem integral da família (2).

Acredita-se que para o desenvolvimento de tais ações educativas em grupos na atenção básica deve-se considerar a realidade dos usuários, suas experiências, vivências e necessidades para construir as temáticas a partir das sugestões dos integrantes do grupo. Dessa forma, o profissional deixa de ser o ator principal e passa a assumir a posição de colaborador. Nesse sentido, a metodologia problematizadora, enfatiza o raciocínio e a reflexão, estimulando a participação na construção do conhecimento (5). Mas para isso, torna-se necessário que os profissionais de saúde tenham conhecimentos sobre a metodologia problematizadora, bem como habilidades e atitudes, além de conhecimentos técnicos do grupo e também sobre o manejo grupal (14).

Os profissionais acreditam que além do aprendizado, a prática grupal possibilita o desenvolvimento da organização do processo de trabalho na unidade. "[...] é uma ação muito positiva tanto para a troca de experiência como para a organização que tentamos adaptar a necessidade do território [...] E aqui, com os funcionários, nós fazemos a troca de experiência sobre a forma como realizou os cuidados com cada paciente".

"Por que, ao trabalhar em SUS na unidade básica, você tem que orientar também. Se você pensar só na escuta psicanalítica e o que tá faltando lá é uma comida, sei lá um básico, essa pessoa precisa ser encaminhada para as assistências e conseguir os recursos que a rede pode oferecer para essa pessoa. Então, por isso, que a gente não trabalha sozinho, né" (Pp7).

A fala dos depoentes reforça o quanto o trabalho grupal é necessário atualmente nos diversos seguimentos, pois contribui para resultados organizacionais, adaptação individual e coletiva (16). As atividades em grupos na Atenção Primária são consideradas estratégias para as práticas assistenciais, constituindo um ambiente coletivo de interação, mas também contribuem para o aprimoramento pessoal e profissional de todos os 
envolvidos através da valorização dos saberes, da cultura e da possibilidade de intervir no processo de saúdedoença de cada pessoa $(19,20)$.

Acredita-se que processo educativo desenvolvido, além da autonomia sobre o controle da saúde, deve-se ter como finalidade o despertar para o empoderamento e a interação de toda a comunidade na realidade dos serviços de saúde (21).

Categoria 3. Dificuldades: pouca adesão e despreparo profissional para desenvolver as atividades de grupo

Os profissionais, ao desenvolverem as atividades grupais, encontram dificuldades como a pouca participação dos usuários. “ [...] eu vejo produtivo, apesar de não ter adesão e a gente sempre tem os mesmos participantes [...]” (Pp1); “[...] não participaram muitas pessoas, vieram em torno de 8 a 11 pessoas somente, sendo que há mais de 300 hipertensos [...]” (Pe2). “[...]. Elas não vêm porque têm que cuidar do filho, do neto e têm muitos compromissos [...]" (Pe4).

A adesão é um conceito amplo, pois envolve a identificação do outro como o dono da sua vida, a identificação de suas carências, sua vulnerabilidade e seu entendimento sobre a doença que o acomete e as propostas ofertadas. Devemos identificar o que os impede de aderirem ao grupo com mais frequência e utilizarem o processo grupal como um espaço de acolhida (22).

Para obter sucesso no trabalho grupal e desenvolver a atividade de forma tranquila e efetiva, Zimerman (10), destaca outros elementos que chama de enquadre ou setting grupal que são, por exemplo, o local, os horários, número de encontros e tempo de duração. É essencial ter um local com boas condições físicas, com boa ventilação, iluminação e com conforto para os participantes (5).

A utilização de outros espaços para realizar atividades educativas é uma forma de otimizar os espaços comunitários e garantir maior participação e troca de experiência entre os envolvidos (23).

Embora os profissionais tenham desenvolvido grupos, pela própria necessidade e/ou pela exigência dos serviços de saúde, eles se sentem despreparados para tal atividade. Referem que, no decorrer da sua formação, o ensino foi centrado no atendimento individual, o que dificulta o desenvolvimento desta atividade e os fazem buscar novos aprendizados. "Eu até nunca tive formação para o grupo, nessa área de grupo; então, sempre foi respondendo ao empregador [...]” (Pp8); “[...] todos os meus estágios ocorreram em forma de atendimento individual. Então, para começar a atender em grupo, eu fui buscar, eu fiz uma formação, uma especialização em M. voltada para grupos [...], a gente foi estudando o sistema SUS e a gente foi vendo a questão da integração de você sair e ir também para a comunidade, de você integrar" (Pp7).

A efetividade do grupo como prática de promoção da saúde pode ser comprometida pela falta de preparo, de planejamento, de capacitação e de conhecimento dos profissionais acerca de como realizar um grupo educativo e todo o processo de dinâmica de grupos (7). Outras dificuldades como a falta de valorização profissional, a sobrecarga de atividades, falta de espaço e condições físicas são também considerados como problema.

Os resultados encontrados nesta pesquisa são corroborados pela literatura, a qual destaca que atividade grupal desenvolvida pelos enfermeiros vem sendo realizadas sem suporte adequado e consistente (24).

Destacam ainda, que a prática de coordenar grupos, seguir metodologias para realizá-los, ter manejo para lidar com cada pessoa do grupo, identificar fatores que interferem improdutivamente no grupo são ações descritas e tarefas vivenciadas por todos profissionais da saúde, mas há profissionais com maior desempenho por ter obtido conhecimento em sua formação curricular, ou porque estudaram e adquiriram habilidades e outros por experimentaram em um dado momento a vivência de estar em grupo. Porém essa prática grupal requer profissional, ambiente e espaço adequado dentro da instituição, materiais disponíveis para desenvolver o trabalho e quando possível realizar em dupla. Embora seja uma proposta do SUS essa prática grupal é preciso discutir sempre sobre o como, quando, porque e onde fazermos para conseguir melhoria individual, material e cultural $(12,13,14,15)$. 


\section{Conclusões finais}

Neste estudo, evidencia-se que os profissionais embora vivenciem a prática grupal apresentam fragilidades quanto a compreensão do conceito de grupo, e em seus relatos aflora o despreparo para realização dessa atividade. Reconhecem a importância do grupo para formação do vínculo entre os atores envolvidos e destacam a atividade grupal como estratégia valiosa para o desenvolvimento das ações educativas em saúde.

Verifica-se que o despreparo dos profissionais pode influenciar negativamente a realização dos grupos, bem como a adesão dos usuários à atividade. Desta forma, fica evidente a necessidade não só do preparo profissional durante a graduação, mas também o desenvolvimento de ações que proporcionem a capacitação daqueles que atuam coordenando as atividades grupais.

As limitações deste estudo estão relacionadas ao número de participantes e ao tipo de abordagem utilizada nesta pesquisa, pois isso limita-se à generalizações dos resultados.

\section{Referências}

1.Ministério da Saúde (BR). Saúde mental. Brasília (DF): Ministério; 2013.

2. Ministério da Saúde (BR). Ações, programas e estratégias. Brasília (DF): Ministério; 2012.

3. Souza LV, Santos MA. Processo grupal e atuação do psicólogo na atenção primária à saúde. J Hum Growth Dev. 2012;22(3):388-95. https://www.dx.doi.org/10.7322/jhgd.46711.

4. Maffacciolli R, Lopes MJM. Os grupos na atenção básica de saúde de Porto Alegre: usos e modos de intervenção terapêutica. Ciênc Saúde Coletiva. 2011;16(Supl. 1):973-82. https://www.dx.doi.org/10.1590/S1413-812320 11000700029.

5. Souza MCMR, Horta NC. Enfermagem em saúde coletiva: teoria e prática. Rio de Janeiro: Guanabara Koogan; 2012.

6. Tiveron JDP, Guanaes-Lorenzi C. Tensões do trabalho com grupos na estratégia saúde da família. Psico [internet]. 2013 [citado 2017 sep 14];44(3):391-401. Disponível em: https://www.revistaseletronicas.pucrs.br/ojs/index. $\mathrm{php} /$ revistapsico/article/view/12200/10414.

7. Silva LD, Beck CLC, Dissen CM, Tavares JP, Budó MLD, Silva HS. O enfermeiro e a educação em saúde: um estudo bibliográfico. Rev Enferm UFSM. 2012;2(2):412-9. https://www.dx.doi.org/10.5902/217976922676.

8. Gomes R. Análise e interpretação de dados de pesquisa qualitativa. Em: Minayo MCS, Deslandes SF, Gomes R, editores. Pesquisa social: teoria, método e criatividade. 32a ed. Petrópolis (RJ): Vozes; 2012. p. 79-108.

9. Bardin L. Análise de conteúdo. São Paulo: Edições 70; 2012.

10. Zimerman DE, Osório LC. Como trabalhamos com grupos. Porto Alegre: Artmed; 1997.

11. Souza DMS, organizador. A prática diária na estratégia saúde da família. Juiz de Fora: UFJF; 2011.

12. Costa RC, Rodrigues CRF. Percepção dos usuários acerca das práticas de promoção da saúde, vivenciadas em grupos, em uma unidade básica de saúde da família. Rev APS [internet]. 2010 [citado 2017 sep 14];13(4):465-75. Disponível em: https://aps.uff.emnuvens.com.br/aps/article/viewFile/836/392

13. Silva G, Iglesias A, Dalbello-Araujo M, Badaró-Moreira MI. Práticas de cuidado integral às pessoas em sofrimento mental na atenção básica. Psicol Cienc Prof. 2017;37(2):404-17. https://www.dx.doi.org/10.1590/1982-3703 001452015.

14. Fortuna CM, Matumoto S, Pereira MJB, Camargo-Borges C, Kawata LS, Mishima SM. Continuing education in the family health strategy: rethinking educational groups. Rev Latino-Am Enferm. 2013;21(4):990-7. https:// www.dx.doi.org/10.1590/S0104-11692013000400022

15. Lucchese R, Calixto BS, Vera I, Paula NI, Veronesi CL, Fernandes CNS. Group teaching in nursing/teaching group nursing practices guided by the Pichon-Rivière theoretical framework. Esc Anna Nery. 2015;19(2):212-9. http s://www.dx.doi.org/10.5935/1414-8145.20150027. 
16. Vieira GLC, Cecílio SG, Torres HC. A percepção dos usuários com diabetes sobre a estratégia de educação em grupos na promoção do autocuidado. Esc Anna Nery. 2017;21(1):e20170017. https:// www.dx.doi.org/10.5935/1414-8145.20170017.

17. Candaten AE, Germani ARM. Educação em saúde: uma proposta educativo-reflexiva na formação do enfermeiro. Rev Enferm [internet]. 2012 [citado 2017 sep 14];8(8):192-207. Disponível em: https://www.revistas.fw.uri.b r/index.php/revistadeenfermagem/article/view/486/887.

18. Luna IT, Silva KL, Dias FLA, Freitas MMC, Vieira NFC, Pinheiro PNC. Ações educativas desenvolvidas por enfermeiros brasileiros com adolescentes vulneráveis às DST/AIDS. Cienc Enferm. 2012;18(1):43-55. https:// www.dx.doi.org/10.4067/S0717-95532012000100005.

19. Bonow CA, Cezar-Vaz MR, SantAnna CF, Cardoso LS, Silva MRS. Limites e possibilidades do desenvolvimento de grupos criativos na Estratégia Saúde da Família. Rev Eletronica Enferm. 2011;13(4):688-94. https://www.d oi.org/10.5216/ree.v13i4.10640

20. Cardoso LS, Cezar-Vaz MR, Silva MRS, Costa VZ. Finalidade do processo comunicacional das atividades em grupo na Estratégia Saúde da Família. Rev Latino-Am Enfermagem. 2011;19(2):396-402. https://www.dx.doi.org/1 $0.1590 /$ S0104-11692011000200023

21. Roecker S, Marcon SS. Educação em saúde na estratégia saúde da família: o significado e a práxis dos enfermeiros. Esc Anna Nery. 2011;15(4):701-709. https://www.dx.doi.org/10.1590/S1414-81452011000400007.

22. Bertolozzi MR, Nichiata LYI, Takahashi RF, Ciosak SI, Hino P, Val LF, Guanillo MCTU, Pereira EG. Os conceitos de vulnerabilidade e adesão na Saúde Coletiva. Rev Esc Enferm USP. 2009;43(spe2):1326-30. https://www.dx .doi.org/10.1590/S0080-62342009000600031.

23. Oliveira MB, Cavalcante EGR, Oliveira DR, Leite CEA, Machado MFAS. Educação em saúde como prática de enfermeiros na Estratégia Saúde da Família. Rev Rene [internet]. 2013 [citado 2017 sep 14]; 14(5): 894-903. Disponível em: https://www.revenf.bvs.br/pdf/rene/v14n5/a04v14n5.pdf.

24. Crispim ZM. Munari DB, Salge AKM, Lucchese R. Atividades grupais na promoção da saúde feminina: revisão integrativa. Rev Rene [internet]. 2011 [citado 2017 sep 14];12(3):636-44. Disponível em: https://www.reven f.bvs.br/pdf/rene/v12n3/26.pdf.

\section{Notas}

*Artigo original de pesquisa

Conflito de interesses: Não existe qualquer conflito de interesses dos autores em relação a este estudo.

Financiamento: Não houve apoio financeiro.

\section{Licencia Creative Commons CC BY 4.0}

Cómo citar: Carvalho VC, Junior AC, Siqueira FPC. Trabalho em grupo: a percepção do profissional do sistema único saúde Investig Enferm Imagen Desarr. 2019;21(1). https://www.doi.org/10.1 1144/Javeriana.ie21-1.tgpp 